\title{
Cardio-Cerebral Infarction Syndrome (CCIS): Definition, Diagnosis, Pathophysiology and Treatment
}

\author{
Mohammed Habib \\ Cardiology Department, Al-Shifa Hospital, Gaza, Palestine
}

Email address:

cardiomohammad@yahoo.com

\section{To cite this article:}

Mohammed Habib. Cardio-Cerebral Infarction Syndrome (CCIS): Definition, Diagnosis, Pathophysiology and Treatment. Cardiology and Cardiovascular Research. Vol. 5, No. 2, 2021, pp. 78-87. doi: 10.11648/j.ccr.20210502.15

Received: May 15, 2021; Accepted: May 29, 2021; Published: June 7, 2021

\begin{abstract}
Acute ischemic stroke (AIS) and coronary artery disease are the major causes of death in Palestine and in the world. Ischemic stroke and acute coronary syndrome have similar vascular risk factors and may evolve as a complication of the respective other disease. The prevalence of coronary artery disease has been reported in one fifth of stroke patients. high incidence rate of acute myocardial infarction (AMI) after recent ischemic stroke and the high risk of acute ischemic stroke after recent myocardial infarction has been reported in several clinical or observational studies. Patients are at increased risk of ischemic stroke following recent myocardial infarction, and aggressive treatment of AMI, including use of reperfusion therapy, decreases the risk of AIS. For patients presenting with AIS in the setting of a recent MI, treatment with alteplase, an intravenous tissue plasminogen activator, can be given, but may be harmful in many conditions. It is important for clinicians to recognize that troponin elevations can occur in the setting of AIS as well as other clinical scenarios and that this may have implications for short- and long-term mortality. So that acute or recent problem in the heart or brain that could result in an acute infarction of the other. In this review we describe the definition and new classification of the cardio-cerebral infarction syndrome with 3 subtypes that reflect the definition, pathophysiology and treatment options.
\end{abstract}

Keywords: Cardio-cerebral Infarction Syndrome (CCIS), Diagnosis, Pathophysiology and Treatment

\section{Introduction}

The incidence of acute ischemic stroke (AIS) after recent myocardial infarction (MI) during the hospital stay ranges from $0.7 \%$ to $2.2 \%$. [1-3] AIS occurred more frequently in the first days after Acute myocardial infarction (AMI), but incidence progressively decreased over time. [4-5] Brandi Witt et al, suggested that during hospitalization for MI 11.1 the AIS occurred per 1000 MI compared with 12.2 at one month and 21.4 at one year. The most positive predictors of ischemic stroke after MI included: older age, hypertension, diabetes, history of previous stroke, history of anterior location MI, previous MI, atrial fibrillation and heart failure [6].

The incidence of AMI after recent ischemic stroke was relatively low and unexpectedly highest during the first year after recent stroke. The 5-year cumulative incidence of AMI was $2.0 \%$. The annual risk was highest in the first year after the index event $1.1 \%$. Coronary heart disease was the most substantial risk factor for AMI after ischemic stroke and conferred an approximate 5 - fold greater risk. [7]

Both AIS and AMI are medical emergency conditions, which require rapid diagnosis and treatment. According to new trial the incidence of AMI patients who diagnosed acute ischemic stroke about $0.009 \%$. [8] In this article we divided cardio-cerebral infarction syndrome into 3 types according to AIS or AMI although diagnostic criteria, pathophysiology and treatment options according to recent clinical trials, metanalysis or case series.

\section{Objectives}

a) Identify the definition and etiologies of cardio-cerebral infarction syndrome.

b) Describe the pathological findings in a patient with each subtype of cardio-cerebral infarction syndrome.

c) Outline the treatment and management options available for patients with each subtype of cardiocerebral infarction syndrome. 


\section{Definition of Cardio-cerebral Infarction Syndrome}

Cardio-cerebral infarction syndrome can generally be defined as Primary disorders (infarction or its complications) of 1 of these 2 organs (Heart or Brain) often result in secondary infarction/injury to the other or to both organs. (figure 1)

\section{Types of Cardio-Cerebral Infarction Syndrome}

The Al-Shifa Hospital Classification of Cardio-Cerebral Infarction Syndrome divided into 3 types (figure 2)

Type I: concurrent cardio-cerebral infarction syndrome: acute myocardial infarction ( $<12$ hours) with acute ischemic stroke $(<4.5$ hours) from last known well or (if patients awake with stroke symptoms or have unclear time of onset $>$ 4.5 hours from last known well, MRI with diffusion-positive FLAIR negative lesions). This type according to causes can be divided into 3 subgroups (Type IA: Cardiac causes, Type IB: Brain Causes, Type IC: Non-cardiac and non- brain causes).

Type 2: Acute ischemic stroke $(<4.5$ hours $)$ from last known well, or (if patients awake with stroke symptoms or have unclear time of onset $>4.5$ hours from last known well, MRI with diffusion-positive FLAIR negative lesions) after recent myocardial infarction (myocardial infarction in the previous 3 months but more than 12 hours).

Type 3: Acute myocardial infarction ( $<12$ hours) after recent ischemic stroke (ischemic stroke in the previous 3 months but more than 4.5 hours).

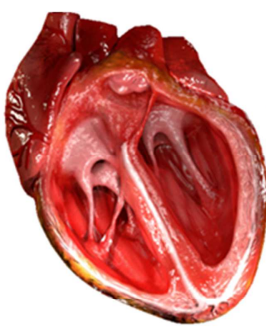

(Acute myocardial infarction) Atrial fibrillation/flutter Intervention: $\mathrm{PCl}$ or $\mathrm{CABG}$ LV systolic dysfunction: $\mathrm{EF}<35 \%$ LV thrombus

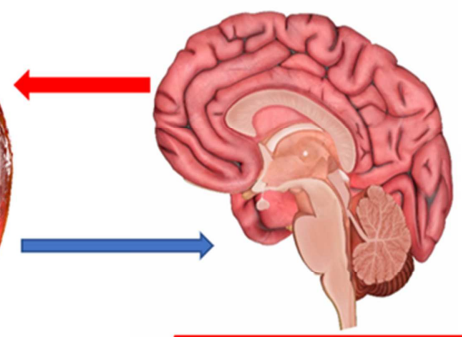

(Acute ischemic stroke) Brain-heart axis Inflammatory cells systemic catecholamines Necrosis- fibrosis

PCI: percutaneous coronary intervention, CABG: coronary artery bypass graft surgery, LV: left ventricle, EF: ejection fraction

Figure 1. Definition of cardio-cerebral infarction syndrome.

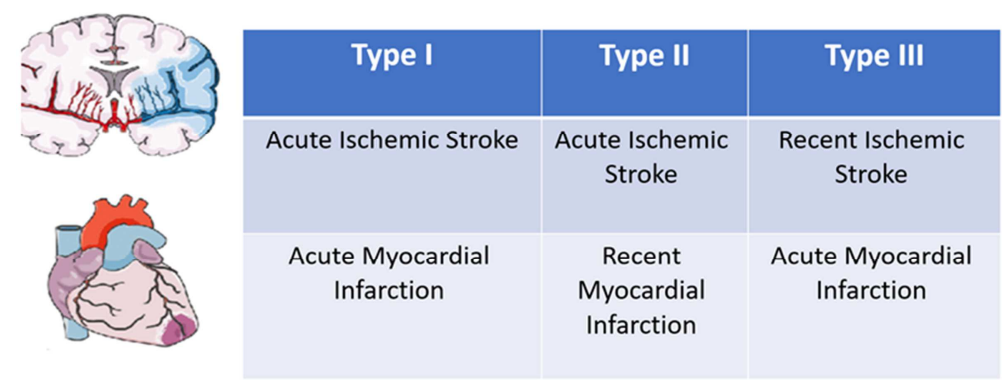

Figure 2. Types of cardio-cerebral infarction syndrome.

The recent ischemic stroke: ischemic stroke in the previous 3 months but more than 4.5 hours or if patients awake with stroke symptoms or have unclear time of onset $>4.5$ hours from last known well, MRI with diffusion-positive FLAIR negative lesions. The recent myocardial infarction: myocardial infarction in the previous 3 months but more than 12 hours

\subsection{Type I: Concurrent Cardio-cerebral Infarction syndrome}

\subsubsection{Definition}

Concurrent cardio-cerebral infarction syndrome can be diagnosed by the presence of synchronous acute onset of focal neurological deficit without intracranial hemorrhage and typical chest pain with evidence of elevation of cardiac enzymes and electrocardiogram changes to confirm myocardial infarction.

\subsubsection{Diagnosis}

Concurrent AIS (a sudden onset of focal neurological deficit caused by an acute cerebrovascular narrowing causes) and AMI (acute elevation cardiac enzyme plus ischemic electrocardiogram and/or symptoms). 


\subsubsection{Pathophysiology}

The pathophysiology of type I cardio-cerebral infarction syndrome can be divided into three categories:

(1) Cardiac causes or Type 1A (table 1)

There are several cardiac causes that lead to concurrent acute stroke with acute myocardial infarction. The most of these is atrial fibrillation can be causes common source of both brain and coronary artery embolism [9]. Acute aortic dissection (type I) with dissection flap extending to coronary arteries and subclavian trunk or common carotid arteries origin had been confirmed to cause concurrent acute myocardial infarction and acute ischemic stroke [10]. In addition, vasospasm due to electrical injury have been reported as an uncommon cause of type I cardio-cerebral infarction syndrome [11].

Pre-existing left ventricular thrombus due to impaired left ventricular ejection fraction or prosthetic valve thrombosis due to low INR ratio can also lead to Type I cardio-cerebral infarction syndrome [12]. Also thrombus formation in the right ventricle in acute right ventricular infarction sitting with right ventricular dysfunction in combination with patent foramen ovale can lead to embolize thrombus for cerebral and coronary territories. Severe hypotension or cardiogenic shock following AMI can also lead to concurrent stroke and myocardial infarction [13].

Table 1. Cardiac causes of type 1 cardio-cerebral infarction syndrome.

\begin{tabular}{ll}
\hline 1 & Atrial fibrillation \\
2 & Type-I acute aortic dissection \\
3 & vasospasm due to electrical injury \\
4 & Pre-existing left ventricle thrombus \\
5 & Right ventricle thrombus formation in acute right ventricular infarction with right ventricular failure in combination with patent foramen ovale \\
6 & can embolize to both vascular territories \\
7 & Severe hypotension/shock \\
8 & Prosthetic valve thrombosis \\
9 & Intracardiac masses (myxoma- papillary fibroelastoma) \\
\hline
\end{tabular}

(2) Brain causes or Type 1B

Brain causes might be an alternative pathophysiology of concurrent cardio-cerebral infarction syndrome. It has been shown that the insular cortex plays a critical role in central autonomic system regulation [14]. Patients with AIS in the parietoinsular region were found to have higher risk of developing atrial fibrillation [15]. An abnormal electrocardiogram, including ST-segment elevation myocardial infarction (STEMI), was found to be related to ischemic stroke in the insular cortex [16]. In addition to electrocardiographic changes, elevated serum cardiac troponin was shown to be associated with acute ischemic stroke in right inferior parietal lobule [17]. Hyperactivation of cardiac sympathetic from an insular cortex lesion can provoke elevation of cardiac enzyme [18]. Results from human studies the right-side stimulation of insular cortex resulted in a predominant sympathetic activation, whereas the left-side stimulation resulted in a predominant parasympathetic effect [19].

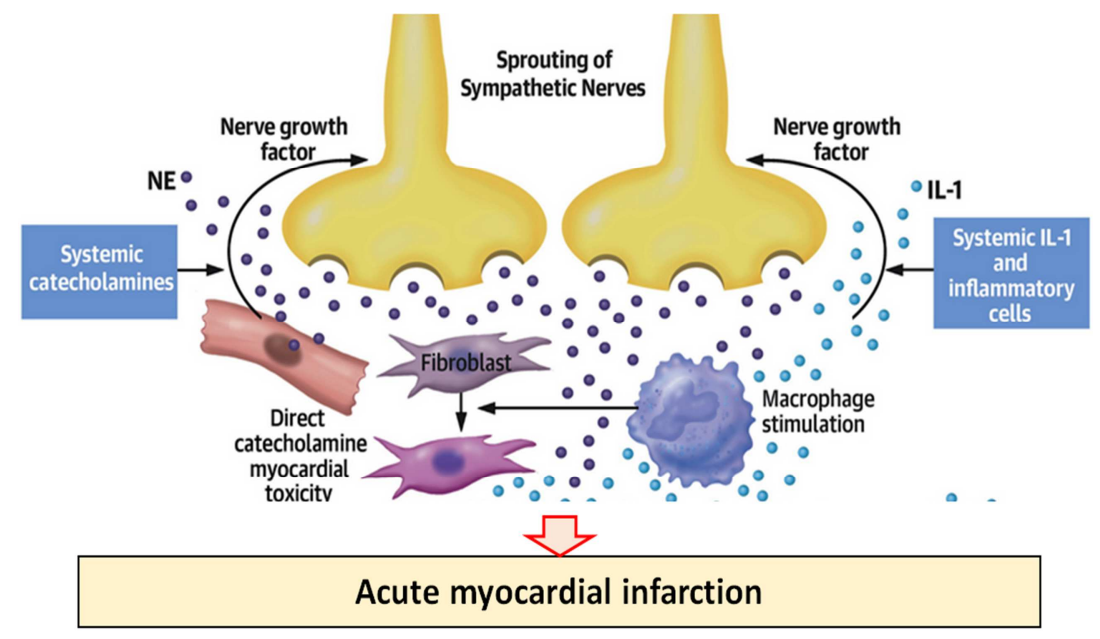

NE norepinephrine, IL Interlaken

Figure 3. Brain causes of type I cardio-cerebral infarction syndrome.

(3) Non cardiac and non-brain causes or Type 1C

Recent studies suggested that coronavirus disease 2019 (COVID-19) infection can be increased the risk of both AIS and AMI. However, the evidence base is limited mainly to case reports and 2 cohort studies. The evidence that COVID19 may increase the risk of acute ischemic cardiovascular events. the underlying mechanisms may cytokine-mediated hypercoagubility and plaque destabilization [20]. Severe hypotension can be causes concurrent infarction in brain and myocardial infarction.

\subsubsection{Treatment}

1. According to the 2018 scientific statement guideline 
from the American Heart Association/American Stroke Association (AHA/ASA), For patients presenting with synchronous AIS and AMI, treatment with IV alteplase (IV- rtPA) at the dose appropriate for acute ischemic stroke, followed by percutaneous coronary intervention (PCI) and stenting if indicated, is reasonable. [20], but no specific recommendation in this guideline for patient with contraindication for thrombolytic in ST Elevation myocardial infarction (STEMI) patients.

2. According to new trial [21] In patients with AIS and concurrent MI, type of MI (STEMI or Non-STEMI) and the time elapsed between the 2 events should be taken in consideration while deciding to deliver IV-rtPA. The concurrent AIS with NSTEMI were not associated with cardiac complications.

We recommended treatment options for type I cardiocerebral infarction syndrome (Figure 4):

1. If patients with STEMI within 12 hours concurrent with AIS within 4.5 hours or (if patients awake with stroke symptoms or have unclear time of onset $>4.5$ hours from last known well, MRI with diffusion-positive FLAIR negative lesions). and no contraindicated for thrombolytic treatment and hemodynamic stable we recommended IV alteplase at the dose appropriate for cerebral ischemia then pharmaco-invasive PCI.

2. In patients with non-STEMI and hemodynamic stable, we recommended IV alteplase and early invasive PCI within 12 hours and if the stroke related to large vessel occlusion (middle cerebral artery or intracranial internal carotid artery) mechanical thrombectomy within 6 hours is recommended.

3. If patients with contraindication for thrombolytic treatment and/or hemodynamic instability we recommended primary PCI for STEMI patients and early invasive strategy for non-STEMI patients. And if the ischemic stroke related to large vessel occlusion mechanical thrombectomy is recommended

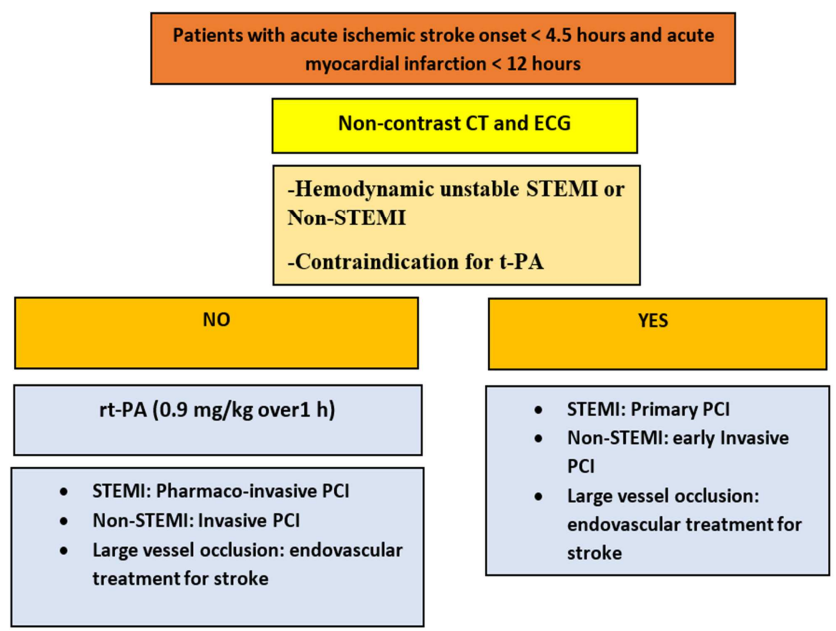

Figure 4. Treatment of type 1 cardio-cerebral infarction syndrome.

PCI: percutaneous coronary intervention, STEMI: ST elevation myocardial infarction, Non-STEMI: non-ST elevation myocardial infarction. ECG: electrocardiogram.

\subsection{Type 2: Acute Ischemic Stroke After Recent Myocardial Infarction}

\subsubsection{Definition}

Acute ischemic stroke in patients with history of recent myocardial infarction in the previous 3 months but more than 12 hours

\subsubsection{Diagnosis}

AIS (a sudden onset of focal neurological deficit caused by an cerebral vascular narrowing cause) and recent history of MI (acute elevation cardiac enzyme plus ischemic electrocardiogram changes and/or symptoms) in the previous 3 months but not in first 12 hours from MI.

\subsubsection{Pathophysiology}

Pathophysiology of type II cardio-cerebral infarction syndrome (table 2):

1. left ventricular mural thrombus (LVMT) due to impaired left ventricle ejection fraction (EF) $<35 \%$ and regional wall motion abnormalities such as dyskinesia or akinesia and septi-apical wall role the most important risk factor. The LVMT is most likely to occur by 2 weeks after an AMI in $0.6-3.7 \%$ of patients [22]. new pharmacological therapy with primary PCI procedures and dual antiplatelet agent, might have contributed to the decreases of LVMT formation after myocardial infarction [23]. Increased coagulation activity during AMI, can potentially lead to increased thrombosis and subsequent thromboembolic events including stroke.

2. The circulatory inflammatory cytokines may be initiated a cascade of events in the cerebral circulation. this phenomenon may contribute to plaque rupture and subsequent thrombus formation in the cerebral circulation [24].

3. Revascularization with early PCI has become the standard of care for patients with acute myocardial infarction and coronary artery bypass graft surgery $(\mathrm{CABG})$ were associated with increased stroke risk. Similarly, analysis of the OASIS [25] registry found that patients with higher rates of invasive cardiac procedures (CABG and $\mathrm{PCI}$ ) suffered from increased risk of ischemic stroke at 6 months $(p=0.004)$.

4. Atrial fibrillation (AF) and atrial flutter after myocardial infarction increased risk of ischemic stroke and occurs in up to $20 \%$ of patients and can cause increased inhospital and long-term mortality [26].

Table 2. Causes of acute ischemic stroke after myocardial infarction.

\begin{tabular}{ll}
\hline 1 & Left ventricle thrombus formation \\
2 & Increased coagulation activity \\
3 & The circulatory inflammatory cytokines \\
4 & Post myocardial infarction atrial fibrillation/ atrial flutter \\
5 & Intervention of myocardial infarction (PCI and CABG) \\
\hline
\end{tabular}

PCI: percutaneous coronary intervention, CABG: coronary artery bypass graft surgery

\subsubsection{Treatment}

According to the 2018 guideline of scientific statement 
from the American Heart.

Association/American Stroke Association (AHA/ASA), [20]:

1. For patients presenting with AIS and a history of recent MI in the past 3 months, treating the ischemic stroke with IV alteplase is reasonable if the recent MI was non-STEMI.(Class IIa)

2. For patients presenting with AIS and a history of recent MI in the past 3 months, treating the ischemic stroke with IV alteplase is reasonable if the recent MI was a STEMI involving the right or inferior myocardium. (Class IIa)

3. For patients presenting with AIS and a history of recent MI in the past 3 months, treating the ischemic stroke with IV alteplase may reasonable if the recent MI was a STEMI involving the left anterior myocardium. (Class IIb)

The main concerns about giving rt-PA to patients with AIS and history of recent MI are (Beyond the bleeding):

1. Thrombolysis-induced myocardial hemorrhage predisposing to myocardial wall rupture

2. Possible ventricular thrombus that could be embolize because of thrombolysis.

3. Post-myocardial infarction pericarditis that may become hemopericardium

The safety of IV rt-PA for acute ischemic stroke (AIS) treatment after recent myocardial infarction (MI) is still controversial. In recent Retrospective review article of 102 AIS patients admitted for AIS with history of recent MI in the previous 3 months. Patients according to treated with standard IV rt-PA dose for AIS were divided into 2 groups: treated or not treated. Four patients with STEMI patients in the week preceding ischemic stroke $(8.5 \%)$ and IV rt-PA treated died from confirmed cardiac rupture/ tamponade. This complication occurred in $1(1.8 \%)$ patients in the nontreated group $(\mathrm{P}=0.178)$, and no non-STEMI patients receiving IV rt-PA had cardiac complications [21].

The new recommendation according to 2021 guidelines of European Stroke Organization (ESO) on intravenous thrombolysis for acute ischemic stroke suggested that [27]:

1. Contraindication of rt-PA For patients with acute ischemic stroke of $<4.5 \mathrm{~h}$ duration and with history of subacute $(>6 \mathrm{~h}$ ) ST elevation myocardial infarction during the last seven days.

2. Insufficient evidence to make a recommendation for patients with acute ischemic stroke of $<4.5 \mathrm{~h}$ duration and with history of ST-elevation myocardial infarction of more than a week to three months.

3. IV rt-PA for patients with acute ischemic stroke of $<4.5$ $\mathrm{h}$ duration and with a history of non-ST-elevation myocardial infarction during the last three months.

The recent retrospective trial among 40396 AIS patients with age $\geq 65$ years, the patients treated with rt-PA were 241 patients $(0.6 \%)$ had recent MI in the past 3 months, of which $19.5 \%$ (41 patients) were ST-segment-elevation myocardial infarction. Patients with recent MI had more severe stroke than those without. Among older patients receiving rt-PA for AIS, a recent history of MI in the past 3 months was associated with higher in-hospital mortality compared with no history of MI in ischemic stroke patients treated with rtPA. This association was more prominent in patients with STEMI than those with NSTEMI. This association was not significant, if the time frame from the onset of MI to the indexed AIS was $>3$ months. [28]

Despite the increasing risk of mortality, further studies are necessary to determine whether the benefit of rt-PA outweighs its risk among AIS patients with a recent history of MI in last 3 months.

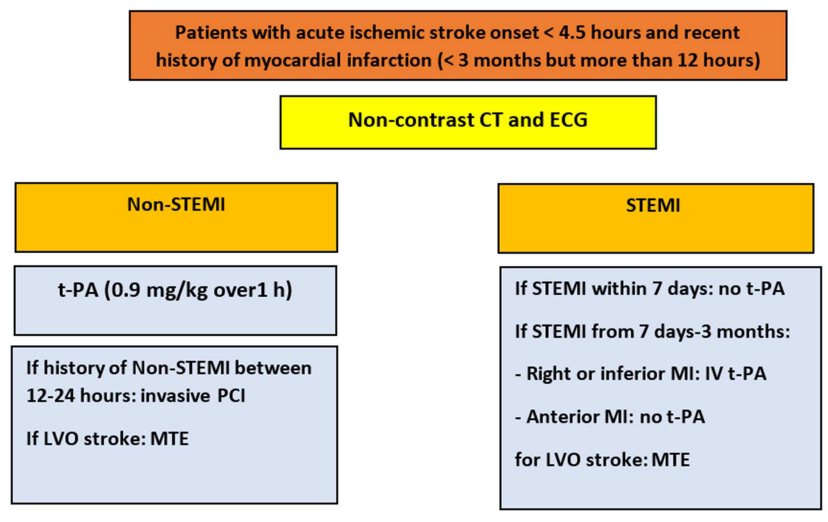

Figure 5. Treatment of type II cardio-cerebral infarction syndrome.

LVO: large vessel occlusion, MTE: mechanical thrombectomy, Non-STEMI: non-ST elevation myocardial infarction, STEMI: ST elevation myocardial infarction, ECG: Electrocardiogram

Thus, we recommended the treatment of type II cardiocerebral infarction syndrome (figure 5):

1. Intravenous rt-PA for patients with acute ischemic stroke of $<4.5 \mathrm{~h}$ duration (if patients awake with stroke symptoms or have unclear time of onset $>4.5$ hours from last known well, MRI with diffusion-positive FLAIR negative lesions). and with a history of NonSTEMI during the last three months.

2. No intravenous rt-PA for patients with acute ischemic stroke of $<4.5 \mathrm{~h}$ duration (if patients awake with stroke symptoms or have unclear time of onset $>4.5$ hours from last known well, MRI with diffusion-positive FLAIR negative lesions). and with history of ST-elevation myocardial infarction of less than one week but more than 12 hours. Mechanical thrombectomy may be a therapeutic alternative in this patient with large vessel occlusion.

3. For patients with acute ischemic stroke of $<4.5 \mathrm{~h}$ duration (if patients awake with stroke symptoms or have unclear time of onset $>4.5$ hours from last known well, MRI with diffusion-positive FLAIR negative lesions). And with history of ST-elevation myocardial infarction of more than one week to three months, there is insufficient evidence to make a recommendation, IV alteplase is reasonable if history of STEMI involving the right or inferior myocardium. But not recommended in patients with history of anterior MI. Mechanical thrombectomy may be a therapeutic alternative in this patients with large vessel occlusion.

4. Anticoagulation with novel oral anticoagulation (such 
as Rivaroxaban) and clopidogrel is recommended in patients with AIS related to cardioembolic causes (left ventricle thrombus and/or atrial fibrillation) and must be at least 3 months then aspirin life long for left ventricle thrombus and 3 months rivaroxaban and clopidogrel then rivaroxaban life long for atrial fibrillation [29].

\subsection{Type 3: Acute Myocardial Infarction After Recent Ischemic Stroke}

\subsubsection{Definition}

AMI in patients with history of AIS in the previous 3 months but not in first 4.5 hours.

\subsubsection{Diagnosis}

AMI (acute elevation of cardiac enzyme plus ischemic electrocardiogram changes and/or symptoms) and history of AIS (a sudden onset of focal neurological deficit caused by cerebral vascular narrowing) in the previous 3 months

\subsubsection{Pathophysiology}

In general, the risk of acute myocardial infarction after ischemic stroke was low. But the most patients with stroke die of heart disease and one in three patients with ischemic stroke without cardiac history have more than $50 \%$ coronary stenosis and about 3\% are at risk of developing myocardial infarction within a year of their stroke. So that patients with stroke need to be screened for silent heart disease and appropriate and aggressive management of total cardiovascular risk factors is required. Notably, patients with history of coronary heart disease showed a 5-fold risk of acute myocardial infarction after stroke onset, and those with cardio-embolism subtype had a higher risk than other subtypes. [7]. Also, poststroke cardiac arrhythmias could be another possible cause of AMI after AIS (table 3).

Table 3. Causes of type III cardio-cerebral infarction syndrome.

Atherosclerotic asymptomatic coronary artery stenosis

Cardiovascular risk factors: dyslipidaemia, hypertension, diabetes

Post stroke arrythmias (atrial fibrillation)

Left ventricle systolic dysfunction or thrombus

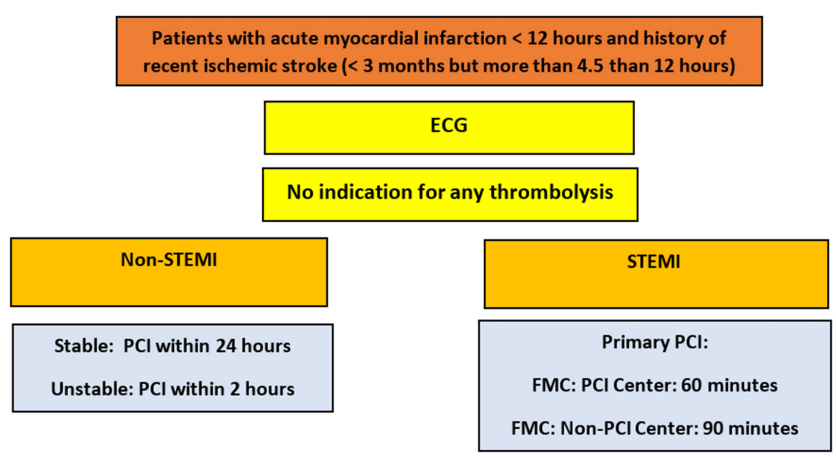

Figure 6. Treatment of type III cardio-cerebral infarction syndrome.

PCI: percutaneous coronary intervention, STEMI: ST elevation myocardial infarction, Non-STEMI: non-STE elevation myocardial infarction, ECG: electrocardiogram, FMC: first medical contact

\subsubsection{Treatment}

Revascularization: the use of thrombolytics is contraindicated and primary PCI for STEMI and early invasive PCI strategy for non-STEMI patient is recommended. (figure 6)

Risk factor modification and treatment such as hypertension, dyslipidemia and diabetes are recommended.

\section{Recommendation of Antithrombotic Therapy in Cardio-cerebral Infarction Syndrome}

The cardioembolic causes treatment must be included novel oral anticoagulation (NOAC) and prefer (Rivaroxaban) or oral anticoagulation OAC (warfarin) and dual or single antiplatelet according to 2020 non-ST elevation acute coronary syndrome guideline of European Society of Cardiology [29]. And to prevention of bleeding in patients with Atrial Fibrillation undergoing PCI trial [30]. In single antiplatelet with (novel) oral anticoagulation $(\mathrm{N}) \mathrm{OAC}$ preference for a clopidogrel over aspirin and prefer NOAC over OAC for the default strategy and in all other scenarios if no contraindications (Prosthetic valve or moderate to severe mitral stenosis). Algorithm for antithrombotic therapy and dosage listed of the following (figure 7):

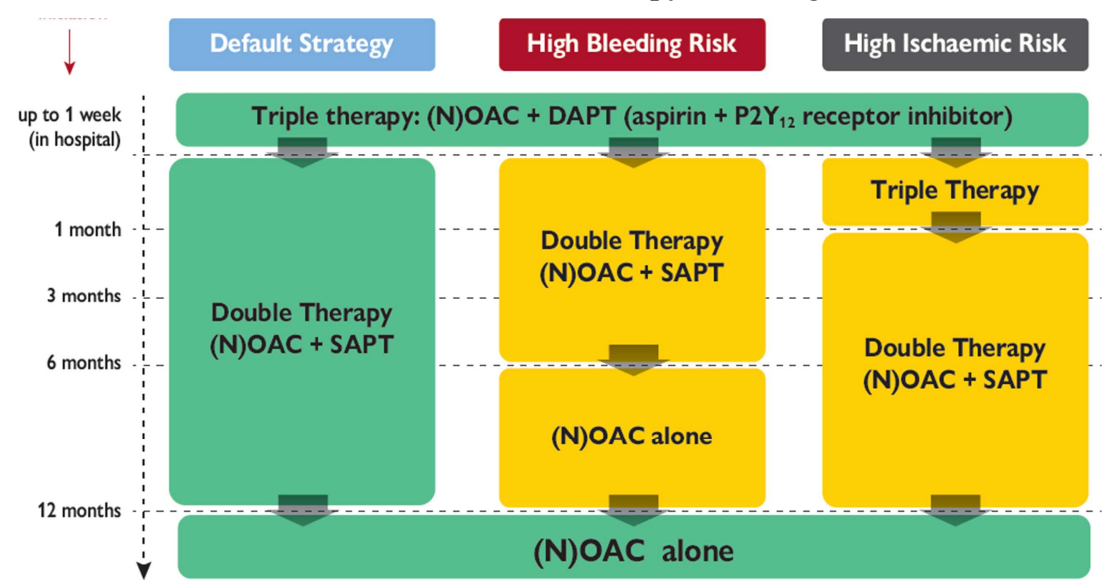

(N)OAC (novel) oral anticoagulation (N), SAPT single antiplatelet therapy, DAPT dual antiplatelet therapy

Figure 7. Algorithm for antithrombotic therapy in patients with cardio-cerebral infarction syndrome and cardioembolic (atrial fibrillation) causes undergoing PCI. 
Triple therapy for one week and must be included: Aspirin $(75-100 \mathrm{mg})+$ Clopidogrel $(75 \mathrm{mg})+(\mathrm{N}) \mathrm{OAC}$ (Rivaroxaban $2.5 \mathrm{mg}$ twice or warfarin: INR $2-3$ and TTR $>70 \%$ ). If patient high risk of thrombosis the duration of triple therapy increase from one week to one month.

Dual therapy preferred included clopidogrel $75 \mathrm{mg}$ daily and (N)OAC and duration 12 months to one year:

AF: (Clopidogrel $(75 \mathrm{mg})+(\mathrm{N})$ OAC (Rivaroxaban $15 \mathrm{mg}$ OD (GFR <60: $10 \mathrm{mg}$ ) or warfarin: INR 2-3 and TTR > 70\%)

LVMT: first 3 months: (Clopidogrel (75 mg)+ OAC (Rivaroxaban $15 \mathrm{mg}$ OD (GFR <60: $10 \mathrm{mg}$ ) or warfarin: INR 2-3 and TTR $>70 \%)$. after 3 months: Aspirin (75-100 mg) + Clopidogrel (75 mg).

If patient high risk of bleeding the duration of dual therapy can be reduce from one year to 6 months.

After one year for lifelong single antiplatelet or (N)OAC: AF: Rivaroxaban or warfarin (Rivaroxaban $20 \mathrm{mg}$ OD (GFR $<60$ : $15 \mathrm{mg}$ ) or warfarin: INR $2-3$ and TTR $>70 \%$ ), LVT: aspirin $100 \mathrm{mg}$ tab once daily. If patient high risk of bleeding start only single antiplatelet or (N)OAC at 6 months for life long.

\section{Initiation of Anticoagulation (N)OAC After Ischemic Stroke}

Patients with a small stroke with National Institutes of health scale score (NIHSS) $<8$ may benefit from early initiation of anticoagulation. But in large ischemic stroke with NIHSS $>8$ initiate of anticoagulation in AF patients between 1 and 12 days after an ischemic stroke, depending on stroke severity.

Initiation of anticoagulation in atrial fibrillation patients after ischemic stroke

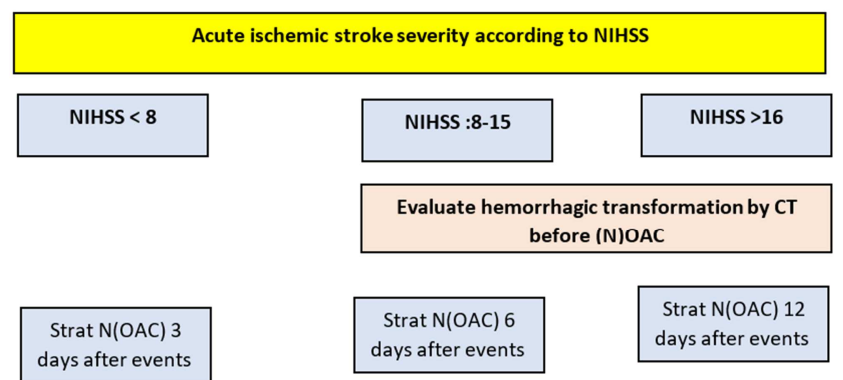

Figure 8. Initiation of anticoagulation in atrial fibrillation patients after ischemic stroke:

NIHSS: National Institutes of health scale score, CT: Computed Tomography (N)OAC: (New) oral anticoagulation

In patient with NIHSS 8-15 anticoagulation initiate 6 days after an ischemic stroke, and if NIHSS $>16$ initiate of anticoagulation must be $>12$ days after ischemic stroke. We suggest repeat brain imaging to determine the optimal initiation of anticoagulation in patients with a large stroke at risk for hemorrhagic transformation. NOACs seem to convey slightly better outcomes, mainly driven by fewer intracranial hemorrhages and hemorrhagic stroke (figure 8).

\section{Recommendations of Lipid-lowering Drugs After Cardio-cerebral Infarction Syndrome [32-35]}

High intensity statins are recommended in all MI and/or AIS patients. The aim of treatment is to reduce LDL-C by $>$ $50 \%$ from baseline and to achieve LDL-C $<1.4 \mathrm{mmol} / \mathrm{L}(<55$ $\mathrm{mg} / \mathrm{dL})$.

If the target LDL-C is not achieved after 4-6 weeks with the maximally tolerated high intensity statin dose, we recommended combination of statin with ezetimibe.

If the target LDL-C is not achieved after 4-6 weeks despite maximally tolerated high intensity statin therapy and ezetimibe, we recommended the addition of a PCSK9 inhibitor to statin and ezetimibe.

\section{Recommendations of (Anti-ischemic / Anti Failure Drugs / Antihypertensive) After Cardio-cerebral Infarction Syndrome}

Angiotensin-converting enzyme (ACE) inhibitors or Angiotensin receptor blocker (ARBs) are recommended in patients with heart failure with reduced LVEF $(<40 \%)$, diabetes, hypertensive or Chronic kidney diseased unless contraindicated (e.g. severe renal impairment, hyperkaliemia, etc.) [36].

Beta-blockers are recommended in patients with prior MI, long-term oral treatment with a beta-blocker should be considered in order to reduce all-cause and cardiovascular mortality and morbidity and in patients with systolic LV dysfunction or heart failure with reduced LVEF $(<40 \%)$. [3741].

Mineralocorticoid receptor antagonist (MRAs) are recommended in patients with heart failure with reduced LVEF $<40 \%$ in to reduce all-cause and cardiovascular mortality and morbidity. [42-43].

\section{Recommendations of Proton Pump Inhibitors in Patients with Cardio-cerebral Infarction Syndrome [44]}

In patients with dual antiplatelet and higher risk of gastrointestinal bleeding:

1. History of gastrointestinal bleeding or ulcer,

2. Corticosteroid use,

3. Oral anti-coagulant therapy,

4. Use of non-steroidal anti-inflammatory drugs, or two or more of 
a. Old age more than 65 years.

b. Gastro-esophageal reflux disease.

c. History of Helicobacter pylori infection.

d. Dyspepsia.

\section{Conclusion}

In type 1 cardio-cerebral infarction syndrome: For patients presenting with concurrent AIS and acute MI, treatment with IV alteplase at the dose appropriate for cerebral ischemia, followed by percutaneous coronary intervention (PCI) and stenting if indicated. But if patient contraindicated to thrombolytic treatment and/or hemodynamic instability we recommended primary PCI for STEMI patients and early invasive strategy for non-STEMI patients. And if the stroke related to large vessel occlusion mechanical thrombectomy is recommended.

In type II cardio-cerebral infarction syndrome: For patients with acute ischemic stroke of $<4.5$ hours duration (if patients awake with stroke symptoms or have unclear time of onset $>$ 4.5 hours from last known well, MRI with diffusion-positive FLAIR negative lesions). And with a history of recent nonST-elevation myocardial infarction during the last three months, we suggest intravenous thrombolysis with alteplase, and Mechanical thrombectomy may be a therapeutic alternative in patients with large vessel occlusion and recent STEMI.

In type III cardio-cerebral infarction syndrome: the use of thrombolytics is contraindicated and primary PCI for STEMI and early invasive PCI strategy for non-STEMI patient is recommended.

\section{Conflict of Interest}

No conflict of interest.

\section{References}

[1] Al Suwaidi J, Al Habib K, Asaad N, Singh R, Hersi A, Al Falaeh H, et al. Immediate and one-year outcome of patients presenting with acute coronary syndrome complicated by stroke: findings from the 2nd Gulf Registry of Acute Coronary Events (Gulf RACE-2). BMC Cardiovasc Disord. 2012; 12: 64 .

[2] Longstreth WT Jr, Litwin PE, Weaver WD. Myocardial infarction, thrombolytic therapy, and stroke. A communitybased study. The MITI Project Group. Stroke. 1993; 24: 587590 .

[3] Kajermo U, Ulvenstam A, Modica A, Jernberg T, Mooe T. Incidence, trends, and predictors of ischemic stroke 30 days after an acute myocardial infarction. Stroke. 2014; 45: 13241330 .

[4] Mooe T, Olofsson BO, Stegmayr B, Eriksson P. Ischemic stroke. Impact of a recent myocardial infarction. Stroke. 1999; 30: $997-1001$.

[5] Brammås A, Jakobsson S, Ulvenstam A, Mooe T. Mortality after ischemic stroke in patients with acute myocardial infarction: predictors and trends over time in Sweden. Stroke. 2013; 44: 3050-3055.

[6] Brandi J. Witt, MD et al. The Incidence of Stroke after Myocardial Infarction: A Meta-Analysis. The American Journal of Medicine (2006) 119, 354. e1-354.

[7] Keon-Joo Lee, MD et al. Five-Year Risk of Acute Myocardial Infarction After Acute Ischemic Stroke in Korea. J Am Heart Assoc. 2021; 10: e018807. DOI: 10.1161/JAHA.120.0188.

[8] Leonard L L Yeo et al. Synchronous cardiocerebral infarction in the era of endovascular therapy: which to treat first? J Thromb Thrombolysis 2017 Jul; 44 (1): 104-111. doi: 10.1007/s11239-017-1484-2.

[9] Tokuda K, Shindo S, Yamada K, Shirakawa M, Uchida K, Horimatsu $\mathrm{T}$, et al. Acute embolic cerebral infarction and coronary artery embolism in a patient with atrial fibrillation caused by similar thrombi. J Stroke Cerebrovasc Dis (2016) 25: 1797-9. doi: 10.1016/j.jstrokecerebrovasdis.2016.01.055.

[10] Nguyen TL, Rajaratnam R. Dissecting out the cause: a case of concurrent acute myocardial infarction and stroke. BMJ Case Rep (2011) 2011: bcr0220113824. doi: 10.1136/bcr.02.2011.382417.

[11] Verma GC, Jain G, Wahid A, Saurabh C, Sharma NK, Pathan $\mathrm{AR}$, et al. Acute ischaemic stroke and acute myocardial infarction occurring together in domestic low-voltage (220$240 \mathrm{~V}$ ) electrical injury: a rare complication. J Assoc Physicians India (2014) 62: 620-3.

[12] Yeo LL, Andersson T, Yee KW, Tan BY, Paliwal P, Gopinathan A, et al. Synchronous cardiocerebral infarction in the era of endovascular therapy: which to treat first? J Thromb Thrombolysis (2017) 44: 104-11. doi: 10.1007/ s11239-0171484-2 11.

[13] Omar HR, Fathy A, Rashad R, Helal E. Concomitant acute right ventricular infarction and ischemic cerebrovascular stroke; possible explanations. Int Arch Med (2010) 3: 25. doi: $10.1186 / 755-7682-3-25$.

[14] Nagai M, Hoshide S, Kario K. The insular cortex and cardiovascular system: a new insight into the brain-heart axis. J Am Soc Hypertens (2010) 4: 174-82. doi: 10.1016/j.jash.2010.05.001.

[15] Vingerhoets F, Bogousslavsky J, Regli F, Van Melle G. Atrial fibrillation after acute stroke. Stroke (1993) 24: 26-30. doi: 10.1161/01.STR.24.1.26.

[16] Christensen H, Boysen G, Christensen AF, Johannesen HH. Insular lesions, ECG abnormalities, and outcome in acute stroke. J Neurol Neurosurg Psychiatry (2005) 76: 269-71. doi: 10.1136/jnnp.2004.037531.

[17] Ay H, Koroshetz WJ, Benner T, Vangel MG, Melinosky C, Arsava EM, et al. Neuroanatomic correlates of stroke-related myocardial injury. Neurology (2006) 66: 1325-9. doi: 10.1212/01.wnl.0000206077.13705.

[18] Cheshire WP Jr, Saper CB. The insular cortex and cardiac response to stroke. Neurology (2006) 66: 1296-7. doi: 10.1212/01.wnl.0000219563.87204.

[19] Daniel Modin, MB et al. Acute COVID-19 and the Incidence of Ischemic Stroke and Acute Myocardial Infarction. Circulation. 2020; 142: 2080-2082. DOI: 10.1161/CIRCULATIONAHA.120.0508. 
[20] Powers WJ, Rabinstein AA, Ackerson T, Adeoye OM, Bambakidis NC, Becker K, et al; American Heart Association Stroke Council. 2018 Guidelines for the early management of patients with acute ischemic stroke: a guideline for healthcare professionals from the American Heart Association/American Stroke Association. Stroke. 2018; 49: e46-e110. doi: 10.1161/STR.0000000000000158.

[21] João Pedro Marto, MD et al. Intravenous Thrombolysis for Acute Ischemic Stroke After Recent Myocardial Infarction Case Series and Systematic Review. (Stroke. 2019; 50: 28132818. DOI: 10.1161/STROKEAHA.119.025630).

[22] Greaves SC, Zhi G, Lee RT, et al: Incidence and natural history of left ventricular thrombus following anterior wall acute myocardial infarction. Am J Cardiol 1997; 80: 442-448.

[23] Motro M, Barbash GI, Hod H, et al: Incidence of left ventricular thrombi formation after thrombolytic therapy with recombinant tissue plasminogen activator, heparin, and aspirin in patients with acute myocardial infarction. Am Heart J 1991; 122: $23-26$.

[24] Lombardo A, Biasucci LM, Lanza GA, et al: inflammation possible link between coronary and carotid plaque instability. Circulation 2004; 109: 3158-3163.

[25] Yusuf S, Flather M, Pogue J, et al: Variations between countries in invasive cardiac proce-dures and outcomes in patients with suspect-ed unstable angina or myocardial infarction without initial ST elevation. OASIS Registry investigators. Lancet 1998; 352: 507-514.

[26] Healey JS, Connolly SJ, Gold MR, Israel CW, Van Gelder IC, Capucci A, et al; ASSERT Investigators. Subclinical atrial fibrillation and the risk of stroke. N Engl J Med. 2012; 366: $120-129$.

[27] Eivind Berge et al. European Stroke Organisation (ESO) guidelines on intravenous thrombolysis for acute ischaemic stroke. European Stroke Journal 2021 http://dx.doi.org/10.1177/2396987321989865.

[28] Taku Inohara, MD, PhD et al. Recent Myocardial Infarction is Associated With Increased Risk in Older Adults With Acute Ischemic Stroke Receiving Thrombolytic Therapy. (J Am Heart Assoc. 2019; 8: e012450. DOI: 10.1161/JAHA.119.012450).

[29] Jean-Philippe Collet, et al. 2020 Acute Coronary Syndromes (ACS) in Patients Presenting without Persistent ST-Segment Elevation (Management of) Guidelines. European Heart Journal (2021) 42, 12891367.

[30] C. Michael Gibson et al. Prevention of Bleeding in Patients with Atrial Fibrillation Undergoing PCI. N Engl J Med 2016; 375: 2423-34.6.

[31] Paulus Kirchhof et al. 2016 ESC Guidelines for the management of atrial fibrillation. European Journal of CardioThoracic Surgery 50 (2016) e1-e8.

[32] Cholesterol Treatment Trialists' (CTT) Collaborators Kearney PM, Blackwell L, Collins R, Keech A, Simes J, Peto R, Armitage J, Baigent C. Efficacy of cholesterollowering therapy in 18,686 people with diabetes in 14 randomisedtrials of statins: a meta-analysis. Lancet 2008; 371: 117125.534 .

[33] Cholesterol Treatment Trialists' (CTT) Collaboration Baigent C, Blackwell L, Emberson J, Holland LE, Reith C, Bhala N,
Peto R, Barnes EH, Keech A, Simes J, Collins R. Efficacy and safety of more intensive lowering of LDL cholesterol: ametaanalysis of data from 170,000 participants in 26 randomised trials. Lancet 2010; 376: 16701681.535.

[34] Sabatine MS, Giugliano RP, Keech AC, Honarpour N, Wiviott SD, Murphy SA, Kuder JF, Wang H, Liu T, Wasserman SM, Sever PS, Pedersen TR, FOURIER Steering Committee and Investigators. Evolocumab and clinical outcomes inpatients with cardiovascular disease. N Engl J Med 2017; 376: 17131722.536.

[35] Flather MD, Yusuf S, Kober L, Pfeffer M, Hall A, Murray G, Torp-Pedersen C, Ball S, Pogue J, Moye L, Braunwald E. Long-term ACE-inhibitor therapy inpatients with heart failure or left-ventricular dysfunction: a systematic over view of data from individual patients. ACE-Inhibitor Myocardial Infarction Collaborative Group. Lancet 2000; 355: 15751581.537.

[36] SOLVD Investigators Yusuf S, Pitt B, Davis CE, Hood WB, Cohn JN. Effect of enalapril on survival in patients with reduced left ventricular ejection fractions and congestive heart failure. N Engl J Med 1991; 325: 293302.538.

[37] Leizorovicz A, Lechat P, Cucherat M, Bugnard F. Bisoprolol for the treatment of chronic heart failure: a meta-analysis on individual data of two placebo-controlled studies CIBIS and CIBIS II. Cardiac Insufficiency Bisoprolol Study. Am Heart J 2002; 143: 301307.540 .

[38] Packer M, Bristow MR, Cohn JN, Colucci WS, Fowler MB, Gilbert EM, Shusterman NH. The effect of carvedilol on morbidity and mortality in patients with chronic heart failure. U.S. Carvedilol Heart Failure Study Group. N Engl JMed 1996; 334: 13491355.541.

[39] Poole-Wilson PA, Swedberg K, Cleland JG, Di Lenarda A, Hanrath P, KomajdaM, Lubsen J, Lutiger B, Metra M, Remme WJ, Torp-Pedersen C, Scherhag A, Skene A, Carvedilol Or Metoprolol European Trial Investigators. Comparison of carvedilol and metoprolol on clinical outcomes in patients with chronic heart failure in the Carvedilol Or Metoprolol European Trial (COMET): randomized controlled trial. Lancet 2003; 362: 713.542.

[40] Kernis SJ, Harjai KJ, Stone GW, Grines LL, Boura JA, O'Neill WW, Grines CL. Does beta-blocker therapy improve clinical outcomes of acute myocardial infarction after successful primary angioplasty? JAmCollCardiol 2004; 43: 17731779.547.

[41] Bangalore S, Makani H, Radford M, Thakur K, Toklu B, Katz SD, DiNicolantonioJJ, Devereaux PJ, Alexander KP, Wetterslev J, Messerli FH. Clinical outcomes with betablockers for myocardial infarction: a meta-analysis of randomized trials. Am J Med 2014; 127: 939953.548.

[42] Pitt B, Zannad F, Remme WJ, Cody R, Castaigne A, Perez A, Palensky J, WittesJ. The effect of spironolactone on morbidity and mortality in patients with severe heart failure. Randomized Aldactone Evaluation Study Investigators. NEngl J Med 1999; 341: 709717.549.

[43] Pitt B, Remme W, Zannad F, Neaton J, Martinez F, Roniker B, Bittman R, Hurley S, Kleiman J, Gatlin M, Eplerenone PostAcute Myocardial Infarction Heart Failure Efficacy and Survival Study Investigators. Eplerenone, a selective aldosterone blocker, in patients with left ventricular dysfunction after myocardial infarction. N Engl J Med 2003; 348: 13091321. 
[44] Valgimigli M, Bueno H, Byrne RA, Collet JP, Costa F, Jeppsson A, Juni P, KastratiA, Kolh P, Mauri L, Montalescot G, Neumann FJ, Petricevic M, Roffi M, Steg PG, Windecker S, Zamorano JL, Levine GN, ESC Scientific Document Group, ESCCommittee for Practice Guidelines (CPG), ESC National Cardiac Societies. 2017 ESC focused update on dual antiplatelet therapy in coronary artery dis-ease developed in collaboration with EACTS: The Task Force for dual antiplatelet therapy in coronary artery disease of the European Society of Cardiology (ESC) and of the European Association for Cardio-Thoracic Surgery (EACTS). Eur Heart J 2018; 39: 213260 . 